\title{
Experimental Optical Generation of DP-16QAM Modulation Format for High Spectral Efficiency Optical Transmission
}

\author{
E. P. Silva ${ }^{(1)}$, L. H. H. Carvalho ${ }^{(1)}$, R. Silva ${ }^{(1)}$, J. P. K Perin ${ }^{(1)}$, M. L. Silva ${ }^{(1)}$, \\ P. P. G. Cardoso ${ }^{(1)}$ and J. C. R. F. Oliveira ${ }^{(1)}$ \\ ${ }^{(1)} \mathrm{CPqD}$ - Centro de Pesquisa e Desenvolvimento em Telecomunicações, Rod. Campinas/Mogi - Mirim, km \\ 118.5,SP - Campinas, Brazil, julioc@cpqd.com.br
}

\begin{abstract}
In this paper, we present a simple experimental method to generate a DP-16QAM optical signal for high spectral efficiency optical transmission. A 16QAM optical signal is generated by passing a DP-QPSK signal through a linear polarization filter in a certain angle so that a square 16QAM can be achieved. Afterwards, we use a polarization multiplexer in order to produce a dual polarization signal. This method proved to be a straightforward and stable way to generate a DP-16QAM signal. In addition, we show that it can provide the same quality in terms of BER per OSNR demonstrated elsewhere.
\end{abstract}

Index Terms - 16QAM, optical transmission, spectral efficiency, modulation formats.

\section{INTRODUCTION}

The increasing demand for telecommunications networks services has been driven mainly by the internet access expansion along the evolution of numerous multimedia applications today available, such as social networks, video on demand and cloud computing [1]. To support this demand, optical backbone networks should continually upgrade their technologies in order to increase its capacity to traffic information efficiently. In this scenario, technologies are being developed to increase optical transmission rates and spectral efficiency [2]. Recently, the Optical Internetworking Forum (OIF) decided to standardize the structure of transmission, reception, mechanical components and modules for $100 \mathrm{G}$ Ethernet, driving its development and commercialization. This standard uses Dual Polarization Quadrature Phase Shift Keying (DP-QPSK) as modulation format, whose spectral efficiency is four times that associated with traditional On-Off Keying format (OOK), for optical transmission at physical layer. This process was a milestone and a paradigm shift in optical transmission technology, due to use of advanced modulation formats, coherent transmission and a considerable number of digital signal processing algorithms to recover optical transmitted information. Research on the application of new modulation formats for higher spectral efficiency transmission was also intensified during recent years, especially formats N-Quadrature Amplitude Modulation (N-QAM) [3], aimed for future generations of optical transmission systems. Systems operating at rates on the order of $200 \mathrm{~Gb} / \mathrm{s}, 400 \mathrm{~Gb} / \mathrm{s}$ and $1 \mathrm{~Tb} / \mathrm{s}$ have been demonstrated at laboratory [4], [5], [6]. However, the discussion of which will be the successor to the $100 \mathrm{G}$ is still open, fueling 
research and development on this technology state of art.

In this article is shown the application of an efficient and low complexity generation of experimental optical coherent modulation format Dual Polarization 16-Quadrature Amplitude Modulation (DP-16QAM) to be used in experiments transmission at rates beyond $100 \mathrm{~Gb} / \mathrm{s}$. It is described analytically the method for obtaining the modulation along with the experimental arrangement used in the laboratory, and are shown and discussed the results.

\section{ANALYTICAL DESCRIPTION OF 16QAM GENERATION METHOD}

Consider the constellation of the QPSK modulation format, as illustrated in Fig.1.

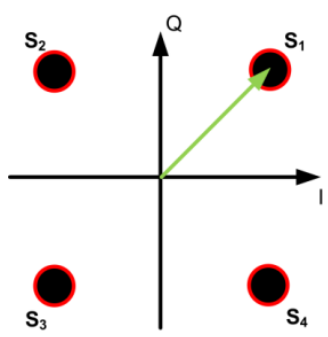

Fig.1: QPSK modulation format constellation diagram.

The symbols $\boldsymbol{S}_{i}$ in Fig.1, with i $\in\{1,2,3,4\}$, can be associated with data points in the complex plane by equation (1). Each symbol combines two bits of information.

$$
\boldsymbol{S}_{i}=e^{j \frac{\pi}{4} * i}
$$

Thus, suppose an optical carrier that has two orthogonal polarization states, $x$ and $y$, each modulated according to a QPSK constellation (DP-QPSK modulation format). Each symbol $\widehat{\boldsymbol{m}}$ of this signal can be represented as the vector equation (2):

$$
\widehat{\boldsymbol{m}}=S_{i, x} \cdot \hat{e}_{x}+S_{i, y} \cdot \hat{e}_{y}
$$

where $\boldsymbol{S}_{\boldsymbol{i}, \boldsymbol{x}}$ and $\boldsymbol{S}_{\boldsymbol{i}, \boldsymbol{y}}$ are identical to those described in (1), $\hat{\boldsymbol{e}}_{\boldsymbol{x}}$ and $\hat{\boldsymbol{e}}_{\boldsymbol{y}}$ are orthonormal vectors $\left(\left\langle\hat{\boldsymbol{e}}_{\boldsymbol{x}} \cdot \hat{\boldsymbol{e}}_{\boldsymbol{y}}\right\rangle=0\right.$ and $\left.\left\langle\hat{\boldsymbol{e}}_{\boldsymbol{x}} \cdot \hat{\boldsymbol{e}}_{\boldsymbol{x}}\right\rangle=\left\langle\hat{\boldsymbol{e}}_{\boldsymbol{y}} \cdot \hat{\boldsymbol{e}}_{\boldsymbol{y}}\right\rangle=1\right)$, each associated with a polarization state of the signal. Given an arbitrary vector $\widehat{\boldsymbol{N}}$ belonging to the plane defined by $\hat{\boldsymbol{e}}_{\boldsymbol{x}}$ and $\hat{\boldsymbol{e}}_{\boldsymbol{y}}$, it can be expressed as (3):

$$
\widehat{\boldsymbol{N}}=\alpha . \hat{\boldsymbol{e}}_{\boldsymbol{x}}+\beta . \hat{\boldsymbol{e}}_{\boldsymbol{y}},
$$

with $\alpha$ and $\beta$ real numbers. The projection $\hat{\boldsymbol{p}}$ of $\widehat{\boldsymbol{m}}$ in the direction $\widehat{\boldsymbol{N}}$ is given by (4):

$$
\begin{gathered}
\widehat{\boldsymbol{p}}=\frac{\langle\widehat{\boldsymbol{m}} \cdot \widehat{\boldsymbol{N}}\rangle}{\langle\widehat{\boldsymbol{N}} \cdot \widehat{\boldsymbol{N}}\rangle} \widehat{\boldsymbol{N}} \\
\widehat{\boldsymbol{p}}=\frac{\left(\alpha \cdot \boldsymbol{S}_{i, \boldsymbol{x}}+\beta \cdot \boldsymbol{S}_{i, y}\right)}{\sqrt{\left(\alpha^{2}+\beta^{2}\right)}} \widehat{\boldsymbol{n}}
\end{gathered}
$$

where $\widehat{\boldsymbol{n}}=\widehat{\boldsymbol{N}} /\|\widehat{\boldsymbol{N}}\|$. Physically, the projection $\hat{\boldsymbol{p}}$ can be seen as the result of passing the modulated carrier through a linear polarizer, whose polarization axis direction is given by $\widehat{\boldsymbol{N}}$. Thus, as seen in (4), 
the coefficient of $\widehat{\boldsymbol{m}}$ projection in the direction $\widehat{\boldsymbol{N}}$ is a linear combination with real coefficients of symbols $\boldsymbol{S}_{\boldsymbol{i}, \boldsymbol{x}}$ and $\boldsymbol{S}_{\boldsymbol{i}, \boldsymbol{y}}$, ie, the complex sum of two QPSK symbols is performed without changing the phase of each individual. Whereas $\boldsymbol{S}_{\boldsymbol{i}, \boldsymbol{x}}$ and $\boldsymbol{S}_{\boldsymbol{i}, \boldsymbol{y}}$, can assume any of four values expressed in (1), using all possible combinations (4) a set of sixteen complex numbers is obtained, corresponding to a constellation formed by weighted superposition of two QPSK constellations. This is the principle which allows 16QAM signal synthetizing.

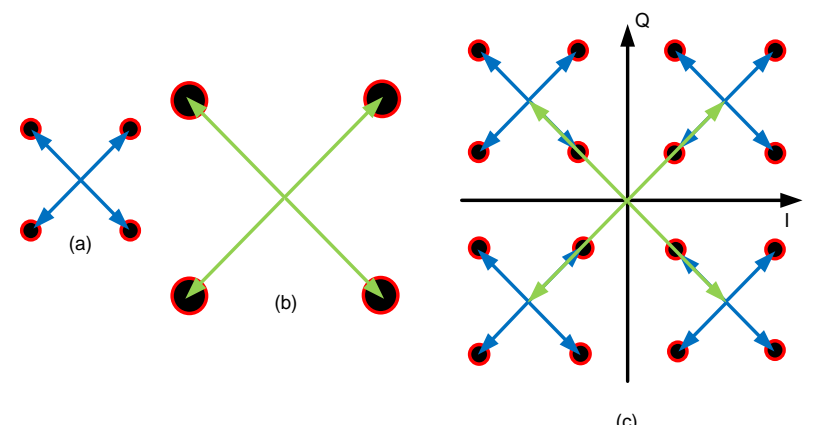

Fig.2: Getting 16QAM constellation from the linear combination of two QPSK constellations. (a) and (b) are scaled QPSK constellations, (c) constellation obtained by superposition of (a) and (b).

Without loss of generality, assume that the polarization state $x$ has the smaller amplitude projection. In Fig.2 (a) we have a graphic illustration of the result of the operation $\alpha . S_{i, x}$ in (4), and Fig.2 (b) the analogous result for $\beta$. $\boldsymbol{S}_{\boldsymbol{i}, \boldsymbol{y}}$. In Fig.2 (c) we have the representation in the complex plane of the symbols generated by the projection $\widehat{\boldsymbol{p}}$ (4), with $\alpha$ and $\beta$ chosen according to a ratio that takes the resulting sixteen points to form a 16QAM constellation. In Fig.2 (c) You can easily see that this relationship is given by $\beta=2 \alpha$. Considering that $\theta$ is the angle between $\widehat{\boldsymbol{N}}$ and $\hat{\boldsymbol{e}}_{\boldsymbol{x}}$, we have:

$$
\begin{gathered}
\cos \theta=\frac{\left\langle\widehat{\boldsymbol{N}} \cdot \hat{\boldsymbol{e}}_{x}\right\rangle}{\|\widehat{\boldsymbol{N}}\| \cdot\left\|\hat{\boldsymbol{e}}_{x}\right\|} \\
\cos \theta=\frac{\alpha}{\sqrt{\left(\alpha^{2}+\beta^{2}\right)}}=\frac{\alpha}{\sqrt{\left(5 \alpha^{2}\right)}}=\frac{1}{\sqrt{5}} \\
\theta=\cos ^{-1}\left(\frac{1}{\sqrt{5}}\right) \approx 63,435^{\circ} \text { or } 26,565^{\circ}
\end{gathered}
$$

Therefore, it is concluded that: given a modulated signal DP-QPSK format, it can be converted to 16QAM with a single polarization state by passing it through a linear polarizer whose angle between the direction of the through slot and the direction of propagation a polarization state of the signal is given by (7). 


\section{THEORETICAL PERFORMANCE OF DP-16QAM MODULATION}

In optical communications, transmissions systems performance can be evaluated with regard to different parameters. Specially, for optical coherent transmission, it's common to analyze the bit error rate (BER) behavior as function of optical signal to noise ratio (OSNR) at receiver's input. It allows several comparisons among systems operating with different modulation formats and carrying distinct loads of information. As the same coherent modulation formats used today in optical transmission were extensively employed in several communications systems, mainly in wireless technologies, their theoretical BER performance with respect receiver's input signal to noise ratio per bit $\left(\mathrm{SNR}_{\mathrm{b}}\right)$ is a well-known topic [7]. Given a modulation format, to define BER curves as a function of OSNR, it's needed only a relationship between OSNR and $\mathrm{SNR}_{\mathrm{b}}$ to convert a BER per $\mathrm{SNR}_{\mathrm{b}}$ known curve in a BER per OSNR curve. As showed in [8], with a reasonable assumption that amplified spontaneous emission (ASE) predominates over other noise sources, the following relation can be established (8):

$$
O S N R=\frac{R_{b}}{2 B_{r e f}} S N R_{b}
$$

where $B_{\text {ref }}$ is the OSNR measure reference bandwidth, commonly taken as $12.5 \mathrm{GHz}(0.1 \mathrm{~nm})$ and $R_{b}$ is the information bit rate, in bits per second. It can be noted that the relation showed in (8) depend only of the information bite rate being transmitted, it's independent if the signal is polarization multiplexed or not. In decibel (dB) values, (8) can be expressed as (9):

$$
\operatorname{OSNR}_{d B}=10 \log _{10}\left(\frac{R_{b}}{2 B_{\text {ref }}}\right)+S N R_{b d B}
$$

Fig.3 shows BER curves of ordinary square 16QAM and optical DP-16QAM, for the specific case of a $224 \mathrm{~Gb} / \mathrm{s}$ transmission $\left(R_{b}=224 \mathrm{~Gb} / \mathrm{s}\right)$, with respect to $\mathrm{SNR}_{\mathrm{b}}(\mathrm{dB})$ and OSNR $(\mathrm{dB})$, respectively.

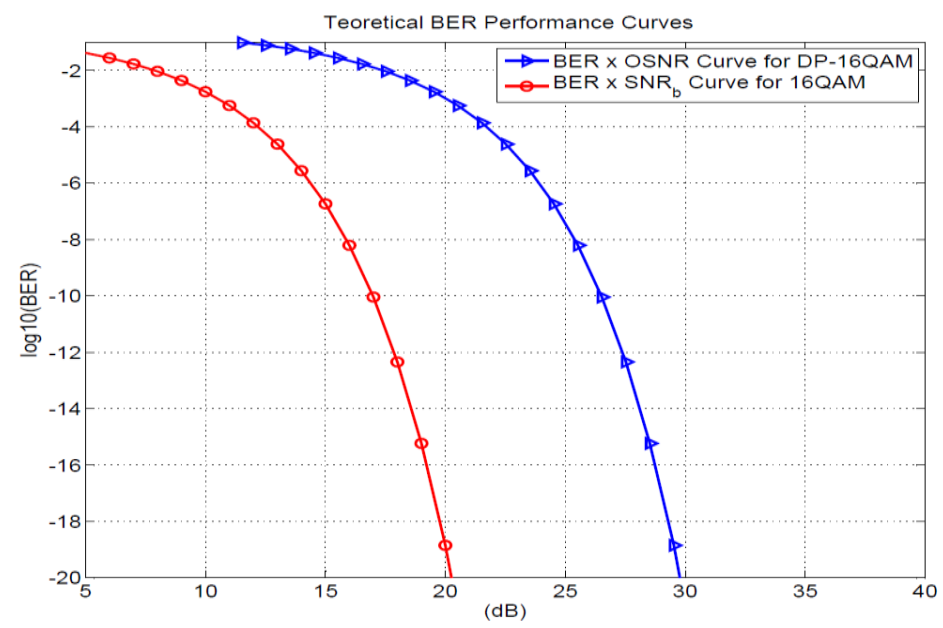

Fig.3: Theoretical BER versus $\mathrm{SNR}_{\mathrm{b}}$ performance curve for ordinary square 16QAM and theoretical BER versus OSNR curve for DP-16QAM at $224 \mathrm{~Gb} / \mathrm{s}$ transmission bit rate. 


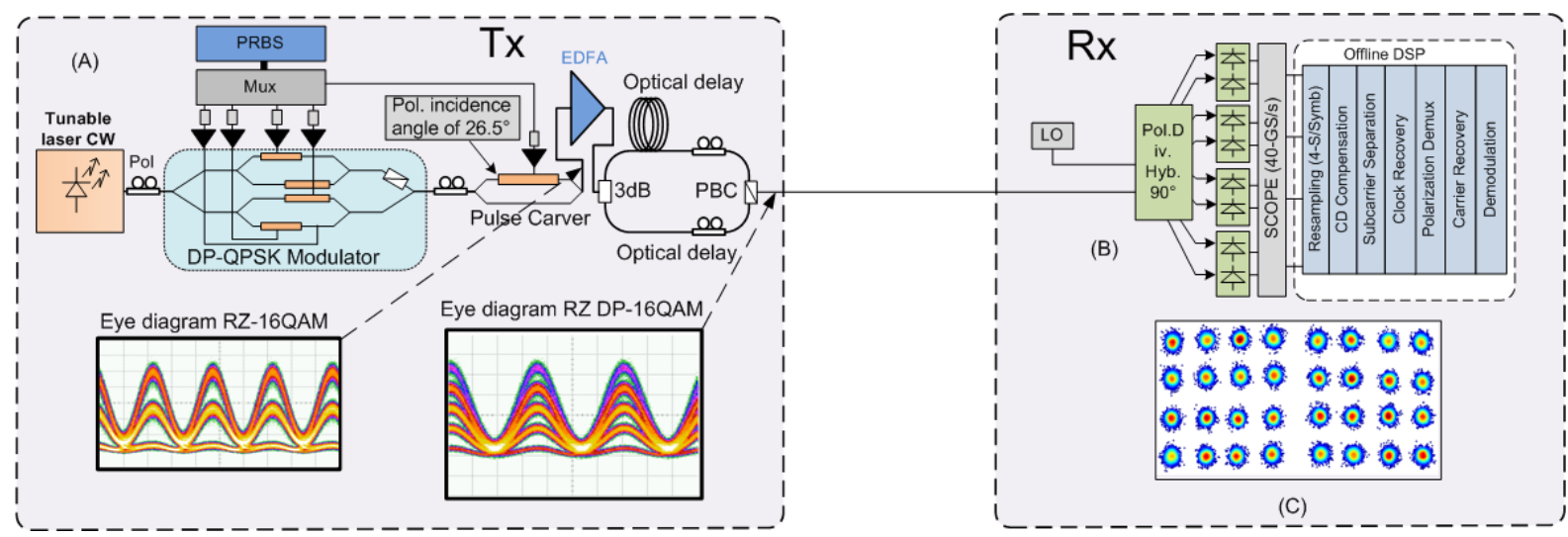

Fig.4: Experimental arrangement to generate a $224 \mathrm{~Gb} / \mathrm{s}$ DP-16QAM signal. (A) Transmitter DP-16QAM, with experimental optical eye diagrams illustrations (B) Coherent receiver with digital signal processing (DSP) blocks and (C) recovered constellations regarding the two polarizations of the signal transmission in a back-to-back configuration.

\section{EXPERIMENTAL SETUP}

The experimental setup to generate a $224 \mathrm{~Gb} / \mathrm{s}$ DP-16QAM signal is shown in Fig.4. A pseudo random bits sequence (PRBS) generator was used to provide information to be transmitted by repeating four pseudo-random binary sequences of length $2^{15}-1$ electrically encoded NRZ signal with a $500 \mathrm{mV}_{\mathrm{pp}}$ (amplitude peak-to-peak), each at a rate of $28 \mathrm{~Gb} / \mathrm{s}$. Each signal was amplified by an $\mathrm{RF}$ driver with $32 \mathrm{GHz}$ bandwidth, so that at the input of the DP-QPSK modulator $\left(\mathrm{V}_{\pi}=3.5 \mathrm{~V}\right)$ the four signals exhibited $7.0 \mathrm{~V}_{\mathrm{pp}}$. A laser with tunable wavelength and linewidth of approximately $100 \mathrm{kHz}$ was used as optical carrier. The modulator acted on the carrier multiplexing the four sequences of $28 \mathrm{~Gb} / \mathrm{s}$ in a modulated DP-QPSK signal with $112 \mathrm{~Gb} / \mathrm{s}, 4$ bits/symbol or 28 GBaud.

The DP-QPSK to 16QAM signal conversion was made in accordance with the principles outlined in Section II. First the DP-QPSK modulator output was directed to a polarization controller and then to amplitude modulator (AM) which acted as pulse formatter or pulse carver in the optical domain. To be driven by a sinusoidal signal with frequency numerically equal to the symbol rate of the signal, the AM acts on each carrier symbol interval, converting the pulse shape from non-return to zero (NRZ) format to return to zero (RZ). Thanks to AM construction characteristics, this device also acts as a linear polarizer. Thus, manually adjusting the input signal polarizations angle via polarization controller a 16QAM can be obtained on its output. Due to practical reasons (easier manual adjustment of the experimental setup), we opted for RZ-16QAM generation. An optical Erbium doped fiber amplifier (EDFA) was used to compensate passive optical devices signal attenuation. The last part of the transmitter is called polarization multiplexing stage. At this stage the incoming signal was split by a $3 \mathrm{~dB}$ coupler. Each signal copy traveled by a different fiber length, in order to make decorrelation between both. One signal copy gets a polarization state rotation of ninety degrees with respect to the second. Both decorrelated copies were coupled by a polarization beam combiner (PBC), passive optical device that can combine two signals with orthogonal polarization states. Then, a RZ DP16QAM signal was exhibited at PBC output. The optical eye diagrams obtained experimentally for 
both RZ-16QAM and RZ DP-16QAM are illustrated in Fig.4.

The optical signal conversion to electrical domain was performed by an optical coherent frontend: local oscillator, $2 \times 8$ hybrid and balanced photodiodes. This structure moves the modulated signal to baseband and linearly converts information from optical domain to electrical domain, demultiplexing polarizations of the signal and separating the components in phase and quadrature of each polarization. The next step is the A/D conversion of the electric signal, carried out at a rate of $40 \mathrm{G}$ samples/s. The digitized signal is processed offline by a set of digital signal processing algorithms to compensate some non-ideal channel effects (chromatic dispersion, polarization mode dispersion, polarization dependent loss, nonlinear effects, imperfection construction of the devices, deviations in frequency between carrier and local oscillator, etc.) [9]. Finally, we obtained the estimated and counted bit error rate, which allow assessing the transmission quality.

\section{RESULTS}

In this section we present the results obtained experimentally by applying the method described in Section II generation. In Fig. 5 the BER back-to-back performance curve as function of measured OSNR at the receiver input is plotted for RZ DP-16QAM as well the theoretical performance curve obtained in section III. In the case of $224 \mathrm{~Gb} / \mathrm{s} \mathrm{RZ} \mathrm{DP-16QAM} \mathrm{signal,} \mathrm{performance} \mathrm{limit} \mathrm{set} \mathrm{by} \mathrm{error}$ correcting codes (FEC limit), considering 7\% redundancy and a 3.8.10-3 BER limit was reached with a measured OSNR value of $20.1 \mathrm{~dB}$. This corresponds to a very small $\sim 1.5 \mathrm{~dB}$ penalty with regard to theoretical curve. Considering the results expressed in [4] and [10], it's observed that performance showed in Fig.5 is comparable, or even better, to that obtained by other 16QAM generation techniques different from that addressed in this paper.

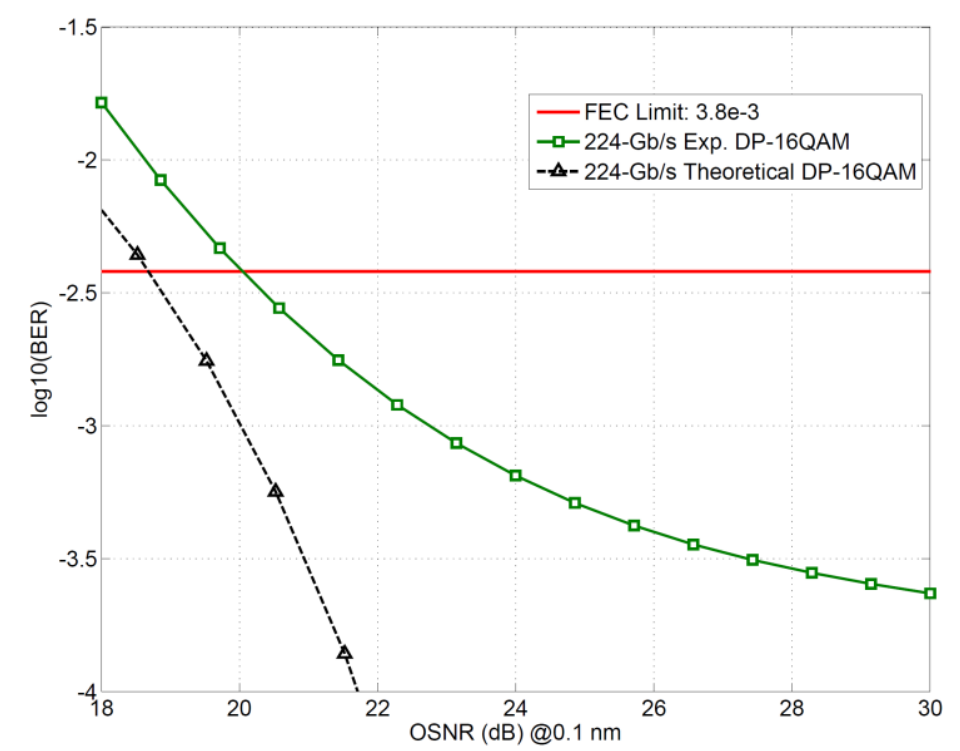

Fig.5: Performance curves of the bit error rate (BER) versus signal-to-noise ratio at the optical receiver input. 
This technique advantages include simplicity of the experimental setup, which requires little modification with respect to that intended for DP-QPSK transmissions laboratory experiments. Due to the project DP-QPSK integrated modulator robustness, there is no need to apply technique phase matching between orthogonally polarized signals arriving at the entrance of MA, as in other forms of alternative generation 16QAM [8]. The disadvantage lies only in the need of incidence angle manual adjustment at MA input. This setup is sensitive to large temperature variations. Fibers that mediate connections between devices must be free from vibrations, to avoid changes in the polarization state arriving at MA input. Fig.6 illustrates an experimental result for a modulated carrier RZ DP-16QAM $224 \mathrm{~Gb} / \mathrm{s}$ transmission over a recirculation loop with a single $72.3 \mathrm{~km}$ optical fiber span (pure silica core fiber with effective area of $80 \mu \mathrm{m}^{2}$ ) and Raman + EDFA amplification. This system achieves a maximum reach at around $720 \mathrm{~km}$.

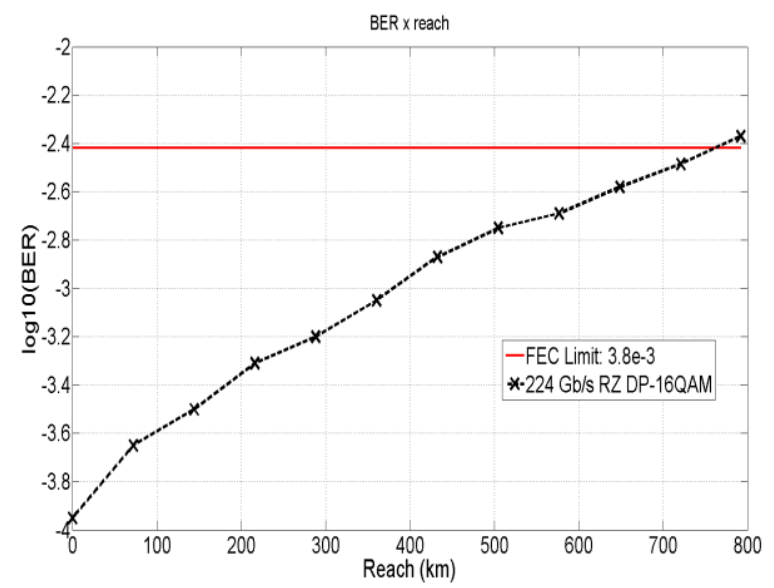

Fig.6: Bit error rate (BER) performance versus distance transmitted for a transmission at $224 \mathrm{~Gb} / \mathrm{s}$ RZ DP-16QAM performed in the laboratory with DP-16QAM generation method described in this paper.

The range of the system can be further improved with a dedicated amplification system design and the use of larger effective area fibers, in order to reduce transmission performance penalties due to fiber nonlinear effects. However, this result expresses well the robustness of the method of experimental generation showed.

\section{CONCLUSION}

In this article it was presented an efficient and low complexity method to implement experimentally the RZ DP-16QAM modulation format for optical transmission at higher rates with high spectral efficiency. The DP-16QAM modulation format is a strong candidate for next generation standard of high rates optical transmission systems. The method proved to be robust, allowing stable transmission experiments with quality. Similar results to those achieved by other approaches to generation and transmission DP-16QAM conventional, but of greater complexity and cost, were demonstrated. The DP-16QAM generation showed enables experimental evaluations without high speed digital/analog converter (Digital-to-Analog Converters - DAC) dependence, only using optical elements to generate 
the 16QAM from DP-QPSK signal. Thus this method allows experimental evaluations of optical transmission systems employing DP-16QAM modulation format, with capacity to operate at rates beyond $100 \mathrm{~Gb} / \mathrm{s}$.

\section{REFERENCES}

[1] Dirk van den Borne, Vincent Sleiffer, Mohammad S. Alfiad, Sander L. Jansen, G. Eason, B., "Towards 400G and beyond: how to design the next generation of ultra-high capacity transmission systems." Opto Electronics And Communications Conference, OECC 2011.

[2] Peter J. Winzer, "Beyond 100G Ethernet", IEEE Communications Magazine, vol. 48, no. 7, pp. 26-30, July 2010. .

[3] René-Jean Essiambre, Gerhard Kramer, Peter J. Winzer, Gerard J. Foschini and Bernhard Goebel, "Capacity Limits of Optical Fiber Networks", Journal of Lightwave Technology, vol. 28, no. 4, February 15, pp. 662-701, 2010.

[4] A. H. Gnauck, P. J. Winzer, S. Chandrasekhar, X. Liu, B. Zhu and D. W. Peckham, "Spectrally Efficient Long-Haul WDM Transmission Using 224-Gb/s Polarization-Multiplexed 16-QAM", journal of lightwave technology, vol. 29, no. 4, pp. 373-377, February 15, 2011.

[5] P. J. Winzer, A. H. Gnauck, S. Chandrasekhar, S. Draving, J. Evangelista and B. Zhu, "Generation and 1,200-km Transmission of 448Gb/s ETDM 56-Gbaud PDM 16-QAM using a Single I/Q Modulator", Optical Communication (ECOC), 2010 36th European Conference and Exhibition.

[6] Schmidt-Langhorst, C.; Ludwig, R.; Hu, H.; Schubert, C. "1 Tb/s Transmission on a Single Wavelength Channel for Future Terabit Ethernet.", Symposium on Photonic Networks, 2009 ITG.

[7] J. G. Proakis, Digital Communications, 4th ed. New York: McGraw-Hill, 2001.

[8] Essiambre, R.-J.; Kramer, G.; Winzer, P.J.; Foschini, G.J.; Goebel, B.; , "Capacity Limits of Optical Fiber Networks," Lightwave Technology, Journal of , vol.28, no.4, pp.662-701, Feb.15, 2010.

[9] Seb J. Savory, "Digital Coherent Optical Receivers: Algorithms and Subsystems.”, IEEE Journal of Selected Topics in Quantum Electronics, vol. 16, no. 5, September/October 2010

[10] Carsten Behrens, Sergejs Makovejs, Robert I. Killey, Seb. J. Savory, Ming Chen, and Polina Bayvel, "Pulse-shaping versus digital backpropagation in 224Gbit/s PDM-16QAM transmission.", Optics Express, Vol. 19, Issue 14, pp. 12879-12884, 2011. 